\title{
Metal-Insulator transition in optical lattice system with site-dependent interactions
}

\author{
Takamitsu Saitou • Akihisa Koga • \\ Atsushi Yamamoto
}

Received: date / Accepted: date

\begin{abstract}
We investigate the half-filled Hubbard model with spatially alternating interactions by means of the two-site dynamical mean-field theory. It is found that a single Mott transition occurs when two kinds of interactions are increased. This implies that the different interactions are essentially irrelevant at the critical point. The nature of the Mott states is also addressed.
\end{abstract}

\section{Introduction}

Ultracold atomic gases have attracted much interest since the successful realization of Bose-Einstein condensation in a bosonic ${ }^{87} \mathrm{Rb}$ system [1]. Due to its high controllability in the interaction strength, particle number, and other parameters, many remarkable phenomena have been observed such as the Mott transitions in the bosonic and fermionic systems [2,3,4]. Recently, the spatial modulation of the interaction has also been realized in the ${ }^{174} \mathrm{Yb}$ gas system [5], which stimulates further theoretical investigations on particle correlations in the ultracold atomic systems.

One of the interesting questions is how the spatial modulation in the interactions affects ground-state properties in the fermionic optical lattice. Although the optical lattice system with uniform interactions has been studied theoretically and experimentally, the system with alternating interactions, which may be one of the simplest systems, has not been discussed so far. Therefore, it is desired to clarify how the alternating interactions affect ground-state properties, in particular, the nature of the Mott transition.

T. Saitou and A. Koga

Department of Physics, Tokyo Institute of Technology, Tokyo 152-8551, Japan

E-mail: koga@phys.titech.ac.jp

A. Yamamoto

RIKEN, Advanced Institute for Computational Science, 7-1-26, Minatojima-minami-machi, Chuo-ku, Kobe, Hyogo 650-0047, Japan 
Motivated by background, we consider the infinite-dimensional Hubbard model with alternating interactions. By means of the two-site dynamical meanfield theory (DMFT) [6], we discuss how particle correlations affect the metalinsulator transition at half-filling. The nature of the Mott phases is also discussed.

The paper is organized as follows. In Sec. 2. we introduce the model Hamiltonian and briefly summarize our theoretical approach. We demonstrate how particle correlations affect ground state properties in Sec. 3. A brief summary is given in the last section.

\section{Model and method}

We study the Hubbard model with alternating interactions, as

$$
H=-t \sum_{\langle i, j\rangle \sigma} c_{i \sigma}^{\dagger} c_{j \sigma}+\sum_{\alpha, i \in \alpha} U_{\alpha}\left(n_{i \uparrow} n_{i \downarrow}-\frac{1}{2}\left[n_{i \uparrow}+n_{i \downarrow}\right]\right),
$$

where $c_{i \sigma}^{\dagger}\left(c_{i \sigma}\right)$ creates (annihilates) a fermion at the $i$-th site with spin $\sigma(=\uparrow, \downarrow$ ). $t$ is the hopping integral, and $U_{\alpha}$ is the site-dependent onsite interaction in the sublattice $\alpha(=A, B)$. At half-filling, the systems with $\left(U_{A}, U_{B}\right),\left(U_{B}, U_{A}\right)$, $\left(-U_{A},-U_{B}\right)$ and $\left(-U_{B},-U_{A}\right)$ are identified since the Hamiltonian is invariant under the particle-hole transformations [7] as $c_{i \uparrow} \rightarrow c_{i \uparrow}$ and $c_{i \downarrow} \rightarrow(-1)^{i} c_{i \downarrow}^{\dagger}$.

To investigate the Hubbard model eq. (1), we make use of DMFT [8,, 9 , $10,11,12$. which has successfully been applied to various strongly correlated electron systems. In the framework of DMFT, the lattice model is mapped to an effective impurity model, where local particle correlations are taken into account precisely. The Green function for the original lattice system is then obtained via self-consistent equations imposed on the impurity problem.

In DMFT, the Green function in the lattice system 13 is given as,

$$
G_{\sigma}(\mathbf{k}, z)=\left[\begin{array}{cc}
z+\mu_{A}-\Sigma_{A \sigma}(z) & -\varepsilon_{\mathbf{k}} \\
-\varepsilon_{\mathbf{k}} & z+\mu_{B}-\Sigma_{B \sigma}(z)
\end{array}\right]^{-1},
$$

where $\mu_{\alpha}$ and $\Sigma_{\alpha}(z)$ are the chemical potential and the self-energy for the $\alpha$-th sublattice, and $\epsilon_{\mathbf{k}}$ is the dispersion relation for the bare band. In terms of the density of states (DOS) $\rho_{0}(x)$, the local Green function is expressed as,

$$
G_{\alpha \sigma}(z)=\int_{-\infty}^{\infty} \frac{\eta_{\bar{\alpha} \sigma}(z)}{\eta_{A \sigma}(z) \eta_{B \sigma}(z)-\varepsilon^{2}} \rho_{0}(\varepsilon) d \varepsilon
$$

where

$$
\eta_{\alpha \sigma}(z)=z+\mu_{\alpha}-\Sigma_{\alpha \sigma}(z)
$$

In the following, we use the semicircular DOS with the half bandwidth $D$, $\rho_{0}(x)=\frac{2}{\pi D} \sqrt{1-(x / D)^{2}}$, which corresponds to the infinite-coordination Bethe 
lattice. Then the self-consistency condition for the sublattice $\alpha$ is represented as,

$$
\mathcal{G}_{0 \alpha \sigma}(z)=z+\mu_{\alpha}-\left(\frac{D}{2}\right)^{2} G_{\bar{\alpha} \sigma}(z)
$$

where $\mathcal{G}_{0 \alpha}$ is the non-interacting Green function of the effective impurity model for the sublattice $\alpha$.

There are various numerical methods to solve the effective impurity problem. To discuss the Mott transitions in the Hubbard model at half-filling, we use here the two-site DMFT method [6], which provides us with a transparent view of the phase transitions [14,15]. In this scheme, we introduce a specific Anderson impurity model for the sublattice $\alpha$, which is connected to only one host site, as

$$
\begin{aligned}
H_{\alpha}^{i m p} & =\varepsilon_{\alpha}^{(d)} \sum_{\sigma} d_{\alpha \sigma}^{\dagger} d_{\alpha \sigma}+\varepsilon_{\alpha}^{(f)} \sum_{\sigma} f_{\alpha \sigma}^{\dagger} f_{\alpha \sigma}+V_{\alpha} \sum_{\sigma}\left(d_{\alpha \sigma}^{\dagger} f_{\alpha \sigma}+\text { H.c. }\right) \\
& +U_{\alpha}\left(n_{f \alpha \uparrow} n_{f \alpha \downarrow}-\frac{1}{2}\left[n_{f \alpha \uparrow}+n_{f \alpha \downarrow}\right]\right)
\end{aligned}
$$

where $d_{\alpha \sigma}\left(f_{\alpha \sigma}\right)$ annihilates a fermion with spin $\sigma$ in the effective bath (localized band) and $n_{f \alpha \sigma}=f_{\alpha \sigma}^{\dagger} f_{\alpha \sigma}$. Note that the effective parameters in the impurity model such as the spectrum of host particles $\varepsilon_{\alpha}^{(d)}$, the energy level of localized band $\varepsilon_{\alpha}^{(f)}$ and the hybridization $V_{\alpha}$, should be determined selfconsistently so that the obtained results properly reproduce the original lattice problem. When the system is half-filling, $\varepsilon_{\alpha}^{(d)}=\varepsilon_{\alpha}^{(f)}=0$.

This simple method provides sensible results as far as low-energy properties are concerned. Namely, for low-energy excitations around the Fermi surface, the Green function for $f$-fermions may be approximated by a single pole as

$$
G_{\alpha \sigma}(z) \sim \frac{w_{\alpha}}{z}
$$

where the residue $w_{\alpha \sigma}$ corresponds to the quasi-particle weight for the sublattice $\alpha$. Therefore, the self-consistency condition is simplified as

$$
V_{\alpha}=\frac{D}{2} \sqrt{w_{\bar{\alpha}}}
$$

By estimating the quasi-particle weight for each Anderson impurity model, we proceed to perform the DMFT iterations. In the following, we discuss the metal-insulator transition in the system with alternating interactions.

\section{Results}

Let us discuss the stability of a metallic state in the Hubbard model with spatially-modulated interactions. By making use of the two-site DMFT method, 
we calculate the quasi-particle weight for each sublattice, which is defined as

$$
z_{\alpha}=\left(1-\frac{d \operatorname{Re} \Sigma_{\alpha}(\omega)}{d \omega}\right)^{-1}
$$

The obtained results for fixed ratios $U_{B} / U_{A}=1.0,2.0,-0.7$ and -2.0 are
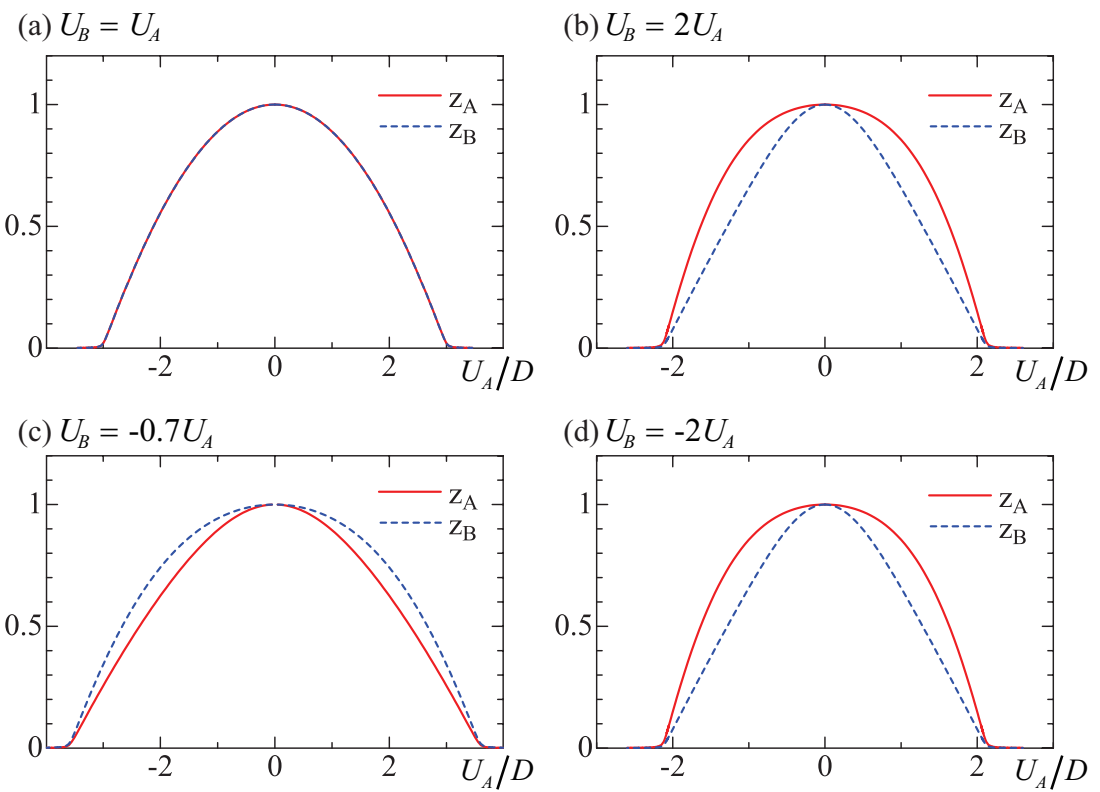

Fig. 1 Solid (dashed) line represent the quasi-particle weight $z_{A}\left(z_{B}\right)$ for the $A(B)$ sublattice obtained in the systems with fixed ratios $U_{B} / U_{A}=1.0$ (a), 2.0 (b), -0.7 (c) and -2.0 (d).

shown in Fig. 1. When $U_{B}=U_{A}$, the system is reduced to the Hubbard model with the homogeneous interactions. As the interactions are introduced, the quasi-particle weights are decreased, as shown in Fig. 1(a). Finally, the quasiparticle weights vanish, where the Mott transition occurs at $U_{A}=3 D$ in the repulsive case and the pairing transition occurs at $U_{A}=-3 D$ in the attractive case. These are consistent with other numerical studies such as the numerical renormalization group $\left(U_{A}=2.94 D\right)[16]$ and the exact diagonalization $\left(U_{A}=\right.$ -2.98D) 17.

When the spatial dependence of interactions is introduced, the quasi-particle weights depend on the sublattices, as shown in Figs. 1 (b), (c) and (d). Namely, the strong renormalization appears in the sublattice with a larger local interaction. Nevertheless, the quasi-particle weights vanish simultaneously in the strong coupling region. This suggests that particle correlations induce a single phase transition. To clarify the nature of the single phase transition, we focus on low energy properties around the Fermi level. When the heavy metallic 
state in the vicinity of the critical point is considered, the quasi-particle weight in the effective Anderson model eq. (6) can be expanded in $V_{\alpha}$ [18, as

$$
w_{\alpha \sigma}=\left(\frac{6 V_{\alpha}}{U_{\alpha}}\right)^{2} .
$$

In this case, the effective hybridization for the other sublattice is comparable since it is given as $V_{\bar{\alpha}}=\frac{3 D}{U_{\alpha}} V_{\alpha}$. Therefore, the fermions in both sublattices are simultaneously renormalized and different interactions are irrelevant around the critical points in this approximation. The critical interactions for the transition are then given as

$$
U_{A} U_{B}=(3 D)^{2}
$$

The phase diagram is shown in Fig. 2. When the interaction is weak, the

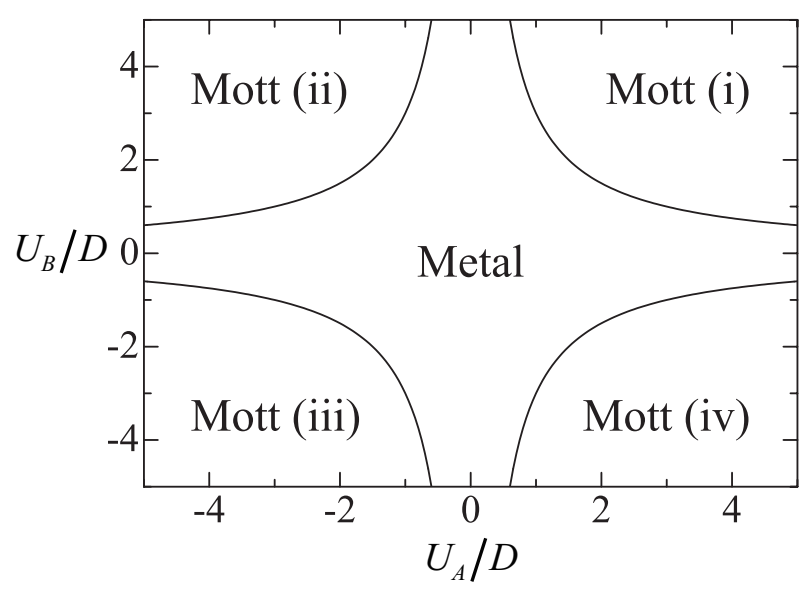

Fig. 2 Ground state phase diagram for the Hubbard model with spatially-modulated interactions.

normal metallic state is realized. On the other hand, when the interactions in both sublattices are large, there exist four kinds of the Mott states, which are denoted as the Mott states (i), (ii), (iii), and (iv), as shown in Fig. 2, We find that the Mott state (i) [(iii)] in the repulsive (attractive) Hubbard model is widely stabilized away from the symmetric limit $\left(U_{A}=U_{B}\right)$. In addition, the Mott states (ii) and (iv) are also stabilized in the system where interactions are alternately arranged repulsive and attractive.

To clarify the nature of these Mott states, we also calculate the double occupancy $D_{i}\left(=\left\langle n_{i \uparrow} n_{i \downarrow}\right\rangle\right)$. In the non-interacting case $\left(U_{A}=U_{B}=0\right)$, the quantity is quarter since the empty, single occupied and doubly occupied states are equally realized at each site. Introducing the interaction, the quantity for each sublattice varies, as shown in Fig. 3. When $U_{B}=2 U_{A}$, the double occupancy 
(a) $U_{B}=U_{A}$

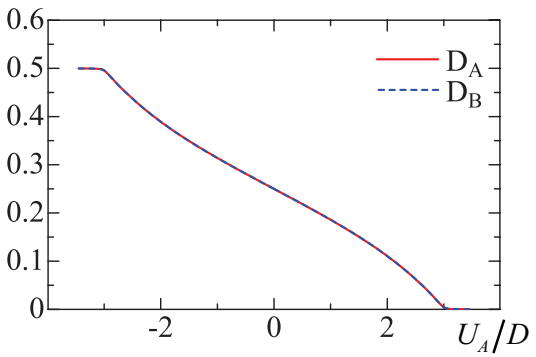

(c) $U_{B}=-0.7 U_{A}$

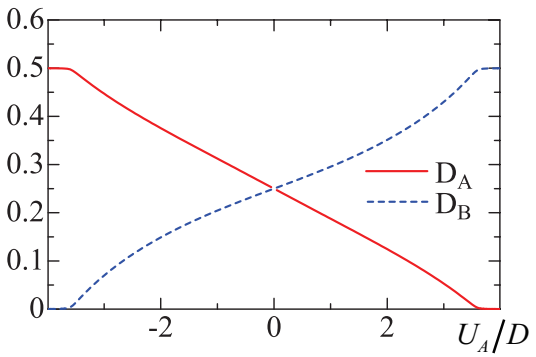

(b) $U_{B}=2 U_{A}$

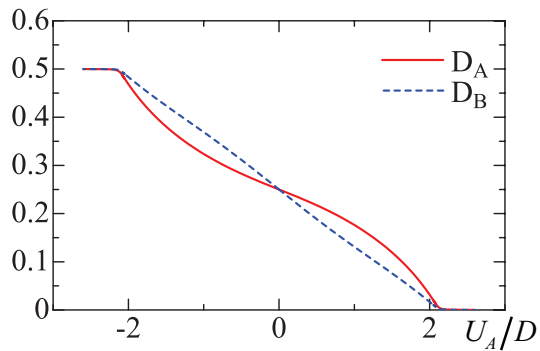

(d) $U_{B}=-2 U_{A}$

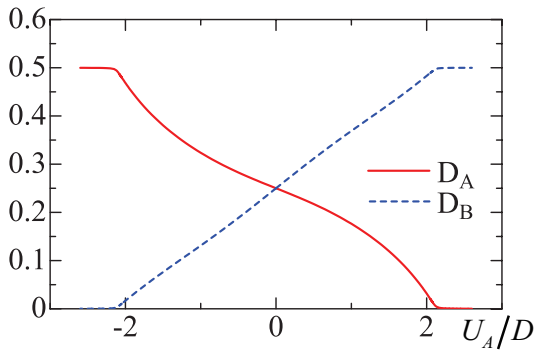

Fig. 3 Solid (dashed) line represent the double occupancy $D_{A}\left(D_{B}\right)$ for the $A(B)$ sublattice obtained in the systems with fixed ratios $U_{B} / U_{A}=1.0$ (a), 2.0 (b), -0.7 (c) and -2.0 (d).

for the sublattice $B$ rapidly decreases since the local Coulomb interaction is large. Finally these quantities for both sublattices vanish simultaneously at the Mott transition point. On the other hand, the double occupancy approaches half as the attractive interaction increases. In this case, the pairing transition occurs at $U_{A}=-2.12 D\left(U_{B}=2 U_{A}\right)$, where the single occupied states are forbidden and the empty or doubly occupied state is realized at each site. It is also found that when the repulsive and attractive interactions alternate in the system $\left(U_{A} U_{B}<0\right)$, the Mott and pairing transitions occur simultaneously in the corresponding sublattices, as shown in Figs. 3 (c) and (d).

In this paper, we have clarified the nature of the Mott transitions in the system with alternating interactions, by restricting our discussions to the paramagnetic case. Although this condition may be relevant for the optical lattice experiments, some ordered states are naively expected at very low temperatures. Therefore, it is necessary to clarify how the magnetically ordered state or the superfluid state is stabilized in the system only with repulsive or attractive interactions $\left(U_{A} U_{B}>0\right)$. On the other hand, the Mott transition should be realized in the system with repulsive and attractive interactions $\left(U_{A} U_{B}<0\right)$ since any ordered states are hard to be stabilized. In the case, the hole doping effect is one of the important problems since the local particle density varies in the optical lattice system with a confining potential. Furthermore, it is expected that particle correlations induce the commensurability in the doped system [19,20], which is now under consideration. 


\section{Summary}

We have investigated the half-filled Hubbard model with spatially-modulated interactions by means of the two-site dynamical mean-field theory. It has been clarified that a single Mott transition occurs when two kinds of the interactions are increased. We have determined the ground state phase diagram and have discussed the nature of these Mott states.

Acknowledgements This work was partly supported by the Grant-in-Aid for Scientific Research 20740194 (A.K.) and the Global COE Program "Nanoscience and Quantum Physics" from the Ministry of Education, Culture, Sports, Science and Technology (MEXT) of Japan.

\section{References}

1. M. H. Anderson, J. R. Ensher, M. R. Matthews, C. E. Wieman, and E. A. Cornell, Observation of Bose-Einstein Condensation in a Dilute Atomic Vapor, Science 269, 198 (1995).

2. M. Greiner, O. Mandel, T. Esslinger, T. W. Hänsch and I. Bloch, Quantum phase transition from a superfluid to a Mott insulator in a gas of ultracold atoms, Nature 415, 39 (2002).

3. R. Jördens, N. Strohmaier, K. Günter, H. Moritz, T. Esslinger, A Mott insulator of fermionic atoms in an optical lattice, Nature 455, 204 (2008).

4. U. Schneider, L. Hackermuller, S. Will, Th. Best, I. Bloch, T. A. Costi, R. W. Helmes, D. Rasch and A. Rosch, Metallic and Insulating Phases of Repulsively Interacting Fermions in a 3D Optical Lattice, Science 322, 1520 (2008).

5. R. Yamazaki, S. Taie, S. Sugawa, and Y. Takahashi, Submicron Spatial Modulation of an Interatomic Interaction in a Bose-Einstein Condensate, Phys. Rev. Lett. 105, 050405 (2010).

6. M. Potthoff, Two-site dynamical mean-field theory, Phys. Rev. B 64, 165114 (2001).

7. H. Shiba, Thermodynamic Properties of the One-Dimensional Half-Filled-Band Hubbard Model. II, Prog. Theor. Phys. 48, 2171 (1972).

8. W. Metzner and D. Vollhardt, Correlated Lattice Fermions in $d=\infty$ Dimensions, Phys. Rev. Lett. 62, 324 (1989).

9. E. Müller-Hartmann, Correlated fermions on a lattice in high dimensions, Z. Phys. B: Condens. Matter 74, 507 (1989).

10. Th. Pruschke, M. Jarrell, and Freericks, Adv. Phys. 44, 187 (1995).

11. A. Georges, G. Kotliar, W. Krauth, and M. J. Rozenberg, Dynamical mean-field theory of strongly correlated fermion systems and the limit of infinite dimensions, Rev. Mod. Phys. 68, 13 (1996).

12. G. Kotliar and D. Vollhardt, Strongly Correlated Materials: Insights From Dynamical Mean-Field Theory, Phys. Today 53, (2004).

13. R. Chitra and G. Kotliar, Dynamical Mean Field Theory of the Antiferromagnetic Metal to Antiferromagnetic Insulator Transition, Phys. Rev. Lett. 83, 2386 (1999).

14. Y. Ono, R. Bulla, and A. C. Hewson, Phase diagram of the Mott transition in a two-band Hubbard model in infinite dimensions, Eur. Phys. J. B 19, 375 (2001).

15. A. Koga, Y. Imai, and N. Kawakami, Stability of a metallic state in the two-orbital Hubbard model, Phys. Rev. B 66, 165107 (2002).

16. R. Bulla, Zero Temperature Metal-Insulator Transition in the Infinite-Dimensional Hubbard Model, Phys. Rev. Lett. 83, 136 (1999).

17. M. Capone, C. Castellani, and M. Grilli, First-Order Pairing Transition and SingleParticle Spectral Function in the Attractive Hubbard Model, Phys. Rev. Lett. 88, 126403 (2002).

18. R. Bulla and M. Potthoff, "Linearized" dynamical mean-field theory for the MottHubbard transition, Eur. Phys. J. B 13, 257 (2000). 
19. A. Koga, N. Kawakami, T. M. Rice, and M. Sigrist, Orbital-Selective Mott Transitions in the Degenerate Hubbard Model, Phys. Rev. Lett. 92, 216402 (2004).

20. K. Inaba and A. Koga, Metal-Insulator Transition in the Two-Orbital Hubbard Model at Fractional Band Fillings: Self-Energy Functional Approach, J. Phys. Soc. Jpn. 76, 094712 (2007) 